\title{
Concepción y el proyecto de ciudad inteligente. Brechas, oportunidades y desafíos.
}

\author{
Concepción Promising smart city Project. Gaps, opportunities and challenges
}

\author{
Herman Durán Urra*
}

Resumen: En la presente investigación se identifican las brechas que tiene la ciudad de Concepción actual en comparación con una smart city futura en relación con la literatura especializada y la agenda nacional que impulsa CORFO. De esta forma, busca explicar el nuevo tipo de desarrollo de ciudades y cómo una nueva gestión territorial impactaría en la vida de sus habitantes. Concepción además de ser recientemente elegida la mejor ciudad para vivir a nivel nacional presenta condiciones necesarias para la implementación de un modelo de ciudad inteligente en ella. Representantes como la directora regional de CORFO y el director de planificación comunal participaron en la investigación entregando su experiencia, diagnóstico y proyecciones en relación con el proyecto de innovación comunal. De la revisión de estas experiencias se ha llegado a la conclusión de que hay ventajas comparativas en Concepción y brechas que obligan a desarrollar procesos para cumplir las condiciones necesarias en la implementación de una ciudad inteligente ellas son: alianzas público-privado, capital social y un gobierno orientado a la innovación.

Palabras claves: Concepción, Alianza público-privado, Capital social, Innovación, Smart cities, TIC's.

\begin{abstract}
This research identifies the gaps in the city of Concepción today compared to a future smart city in relation to with the specialized literature and national agenda that drives CORFO. In this way, it seeks to explain the new type of development of cities and how a new territorial management would impact the lives of its inhabitants. Conception in addition to being recently chosen as the best city to live nationally, presents necessary conditions for the implementation of a smart city model in it. Representatives such as CORFO's director and director of community planning participated in the investigation delivering their experience, diagnosis and projections in relationship with the community innovation project. The review of these experiences has concluded that there are comparative advantages in Concepción and gaps that require the development of meet the necessary conditions in the implementation of a smart city are: public-private partnerships, social capital and innovationoriented government.
\end{abstract}

Keywords: Concepción, public-private partnerships, social capital, Innovation, Smart cities, TIC's

Recibido: 2 julio 2020 Aceptado: 24 agosto 2020

\footnotetext{
* Chileno, Licenciado en Ciencia Política y Administración Pública, Coordinador Diálogo Naciones Unidas, hduran2016@udec.cl Agradezco al profesor Sergio Toro Maureira, ya que incluso en tiempos de turbulencia y de estallido, conté siempre con su incondicional apoyo y guía.
} 


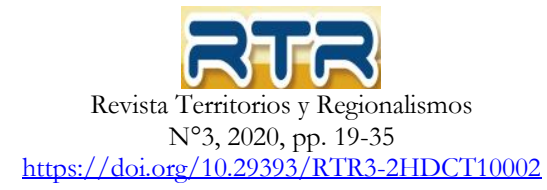

\section{Introducción}

Las ciudades de América Latina y el Caribe viven uno de los procesos de crecimiento demográfico más significativos que ha vivido el planeta (BID, 2016). Para el 2015, el 80\% de la población de América Latina y el Caribe ya estaba viviendo en ciudades (CEPAL, 2019), y en Chile esa cifra aumentó a un 87,8\%, según datos expuestos por Censo Poblacional 2017 realizado ese año (CENSO, 2018).

Lo anterior, se incluye a los desafíos expuestos por el cambio climático y el surgimiento de nuevas tecnologías como el internet de las cosas, la inteligencia artificial y el Big Data, además de la consolidación de economías circulares y colaborativas, acompañado de las nuevas demandas sociales, fuerza a replantear los paradigmas con los cuales se ha venido construyendo la ciudad.

Se espera que para el 2050 el 70\% de la población mundial viva en ciudades (ONU, 2018). Cumpliendo un rol protagónico de los acontecimientos sociales, políticos, ambientales y económicos que se presentan, se espera que las urbes sean capaces de ofrecer las mejores condiciones para el desarrollo integral, inclusivo y sustentable de sus habitantes.

Bajo estas circunstancias, Chile no se queda atrás y se posiciona como líder en Latinoamérica en dar soluciones innovadoras y tecnológicas (Cohen \& Obediente, 2014). Ya se encuentra asentada la idea de que este tipo de soluciones están abriendo oportunidades para ciudades alrededor del mundo ${ }^{1}$, lo que les permite ser más eficientes, mejorar sus economías, bajar su impacto al medio ambiente y, a la vez, mejorar la calidad de vida de sus ciudadanos.

Lo anterior, se adecúa en la búsqueda de implementar un modelo de desarrollo de smart city por medio de iniciativas de colaboración, innovadoras y tecnológicas, para las ciudades del territorio nacional, que abren espacio a nuevas oportunidades de crecimiento, respetando el medio ambiente y, sobre todo, en función de mejorar la calidad de vida de sus habitantes.

A estos nuevos modelos de desarrollo de ciudades se les define como ciudades inteligentes (smart cities) (Cohen \& Obediente, 2014).

Actualmente no se cuenta con una definición universalmente aceptada sobre qué es una smart city (SC). De acuerdo con Caragliu et al (2011) la constitución de las "ciudades inteligentes" se asocia al impacto de las tecnologías de información y comunicación (TICs) y la expansión de su infraestructura.

Otros autores le dan énfasis a que es un territorio con gran capacidad de aprendizaje e innovación, creativo, con presencia de instituciones de investigación y desarrollo, centros de formación superior, dotado con una infraestructura digital y TICs, junto a un elevado nivel de rendimiento de gestión (Sikora - Fernández, 2017).

Chile comenzó a incursionar en las "ciudades inteligentes" y está impulsando diversas iniciativas al respecto. Entre éstas, se encuentra: Agenda Digital 2020, creación de la Mesa Institucional de Smart Cities y Mesa Técnica de Smart Cities, ambos organizadas por Fundación País Digital y Cepal (Cohen \& Obediente, 2014); y el Programa Estratégico Regional: Santiago Ciudad Inteligente (CORFO, 2018).

En comparación con el resto de América Latina, su capital: Santiago de Chile, es la ciudad más inteligente de acuerdo con el Latam Smart City Awards (CORFO, 2019). Y según el índice IESE Cities in Motion (Berrone \& Enric, 2019), Santiago es la ciudad más smart de América Latina y 66 a nivel global.

En consecuencia, a los buenos resultados del plan de CORFO para crear ciudades inteligentes, este será expandido y llegará a otras 5 ciudades, que serán: Antofagasta, Valparaíso, Temuco, Nuble y Concepción.

De esta forma, la ciudad de Concepción es candidata a ser una smart city, ya que, posee algunas de las dimensiones fundamentales para ser considerada una ciudad inteligente, estas serán detalladas posteriormente.

1 Ámsterdam, Singapur, Boston, San Francisco, Estocolmo y Rio de Janeiro, por mencionar "las ciudades más importantes", términos de éxito e inteligencia (Ching, 2013). 


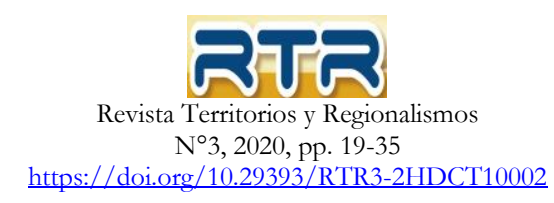

Esta investigación aspira a demostrar las brechas que tiene que superar la ciudad de Concepción, recientemente nombrada como "la mejor ciudad para vivir"' y candidata a convertirse en una smart city nacional, para llegar a ser una ciudad inteligente, en base a la literatura especializada e informantes claves que fueron consultados.

Representantes de la ciudad que están relacionados con la temática central de esta investigación compartieron su opinión y proyecciones en base a su experiencia y área de desempeño laboral.

De esta forma la directora regional de CORFO, el Director de Planificación Urbana comunal y el Director de Desarrollo e Innovación de la Universidad de Concepción, por mencionar algunos, entregaron su opinión y proyección de como la ciudad de Concepción debe guiar su desarrollo para llegar a ser una ciudad inteligente.

En base a la literatura especializada, el proyecto estratégico nacional impulsado por CORFO y la opinión de los informantes clave consultados para esta investigación, las condiciones que debe presentar la ciudad de Concepción para convertirse en una smart city son: capital social, Alianzas público - privada y un gobierno orientado a la innovación. Lo que este investigador denominó como "triángulo smart"

Las partes de esta investigación son una introducción donde se expone la tensión detectada, problematización y solución de esta. Se da paso a una sección de definiciones de smart city, dimensiones y contraste comunal en el ranking nacional de ciudades inteligente. Se presentan y definen las puntas del triángulo smart. Para concluir con el camino a seguir, propuesta y reflexiones finales sobre Concepción, Ciudad Inteligente.

\section{Smart Cities: definiciones, dimensiones y contraste comunal en ranking nacional.}

Actualmente no se cuenta con una definición universalmente aceptada sobre qué es una smart city (SC), se le califica de novedoso, aunque confuso y no bien definido. De acuerdo con Caragliu et al (2011) la constitución de las "ciudades inteligentes" se asocia al impacto de las tecnologías de información y comunicación (TICs) y la expansión de su infraestructura. Odendaal (2003) complementa que estas ciudades sacan provecho de las oportunidades que ofrecen las TICs en la promoción de la prosperidad e impulsar las economías basadas en el conocimiento como factor productivo.

Se señala también pueden ser consideradas como ciudades que por medio de inversiones en capital humano, social, infraestructuras de transporte y TICs contribuyen al desarrollo económico sostenible, con una gestión racional de los recursos naturales, a través de un gobierno participativo (VillarejoGalende, 2015) y esto con el objetivo de que las personas mejoren su calidad de vida (Cohen \& Obediente, 2014); habiendo otros que dan énfasis a que corresponde a un territorio con gran capacidad de aprendizaje e innovación, creativo, con presencia de instituciones de investigación y desarrollo, centros de formación superior, dotado con una infraestructura digital y TICs, junto a un elevado nivel de rendimiento de gestión (Sikora - Fernández, 2017).

En primer lugar, todas ellas hablan de innovación, creación y alianzas entre los sectores públicoprivado y la academia. De igual forma, la diferencia se centra en el grado de importancia en el uso de TICs en su forma de gobierno y diario vivir.

La primera de ellas se centra en que este nuevo tipo de ciudades puede ser considerada como un territorio con gran capacidad de aprendizaje e innovación, creativo con presencias de instituciones de investigación y desarrollo, centros de formación superior, dotado con infraestructura digital y tecnologías de comunicación, junto con un elevado nivel de rendimiento de gestión.

Dando gran importancia a que estas presenten capital humano y social, infraestructura de comunicaciones, tanto tradicional como moderna (transporte y tecnologías de comunicación, respectivamente) y su desarrollo debe ajustarse a la teoría del desarrollo sostenible. Asimismo, la

2 Atlas de Bienestar Territorial, Universidad Adolfo Ibáñez, Chile, 2019. 


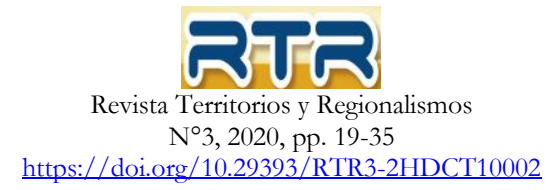

participación ciudadana en su sistema de gobierno debe contribuir a la mejora de calidad de vida de las personas (Smart Governance) (Sikora - Fernández, 2017).

Luego, otro concepto, el cual, hace referencia a que la nueva forma de desarrollo de ciudad tiene un vínculo directo con el uso extensivo y eficiente de las TICs, dirigidas a mejorar la calidad de vida de la población, lo cual, necesariamente tendría que conllevar a un mayor cuidado al medio ambiente y la reducción de la desigualdad social.

Agregando esta nueva forma de pensar, la ciudad es compatible con el concepto de innovación inclusiva, el cual plantea la necesidad de que los beneficios de la innovación sean en beneficio principalmente de los sectores menos favorecidos de la población (Alvarado López, 2018).

De las misma forma para Caragliu, Del Bo y Nijkamp (2011) el concepto de ciudad inteligente se asocia a la discusión y debate relacionados a las estrategias y condiciones que potencia la evolución de las urbes a ecosistemas de innovación, abiertos y sostenibles. Entendiendo así la smart city no como una noción estática sino como un escenario futuro, en que la ciudadanía, por medio de la tecnología, colaboran en configurar el desarrollo urbano.

Para el objetivo último de esta investigación se escogió la definición de Sikora-Fernández (2017), pues a juicio de este investigador es la más completa, esto ya que considera todos los aspectos necesarios para que una ciudad llegue a ser considerada smart. Las cuales, por aplicación de tecnologías, en sus diferentes ámbitos, se transforman en localidades más eficientes en el uso de sus recursos; siendo el conocimiento un factor productivo indispensable en su avance y el desarrollo sostenible su estrategia productiva de crecimiento económico.

En relación con las definiciones presentadas. Las cuatro resaltan el uso de las TICs como importante factor de desarrollo de las ciudades inteligentes.

Ahora, en lo que varían es en el grado de importancia que esta tiene para el autor, mientras que Alvarado (2018) hace referencia directa a que estas están en función de mejorar la calidad de vida de las personas, para Cohen (2014), estas toman un papel multifacético y más dinámico, dando justamente este tipo de perfil a la localidad en la que se implemente; en lo que están en plena sintonía, es que este nuevo tipo de desarrollo de ciudad debe estar acorde a la teoría del desarrollo sustentable, además, de que sea un aporte en dar solución a los principales problemas a los que se ven enfrentados los ciudadanos.

De acuerdo con Sikora-Fernández (2017) y la Comisión de Ciudades Digitales y del Conocimiento (2012), el concepto de smart city está compuesto por las siguientes dimensiones:

1) Economía (Smart economy): Hace referencia a que las ciudades se comprometen a destacar por una elevada productividad, apoyada en la penetración del uso de las TICs por parte de las empresas, la promoción de la iniciativa emprendedora, como en la retención y atracción de talento y fomento de la creatividad, en directa relación con la "economía basada en el conocimiento" (Odendaal, 2003).

2) Transporte y comunicación (Smart mobility): En consecuencia, del uso de las TICs las urbes toman forma de una enorme red de conexiones de alta velocidad. Así deben ser capaces de asegurar la conectividad e infraestructura de transporte y comunicación, tanto tradicional como moderna, y a la vez de asegurar un acceso público a internet para todos sus habitantes.

3) Medioambiente (Smart enviroment): La smart city da uso eficiente y optimiza el consumo de energía, ya que su gestión de recursos se basa en el concepto de desarrollo sostenible. Conforme al objetivo de "La Agenda 2030 para el Desarrollo Sostenible"3 número 11, ya que esta pretende lograr que la ciudades y los asentamientos humanos sean inclusivos, seguros, resilientes y sostenibles (Naciones Unidas, 2016).

\footnotetext{
${ }^{3}$ La agenda 2030 para el Desarrollo Sostenible, que se constituye en base a 17 objetivos de acción, fue aprobada en septiembre de 2015 por la Asamblea General de las Naciones Unidas, y establece una ruta hacia la sostenibilidad económica, social y ambiental de los 193 Estados miembros que la suscribieron; entre ellos Chile.
} 


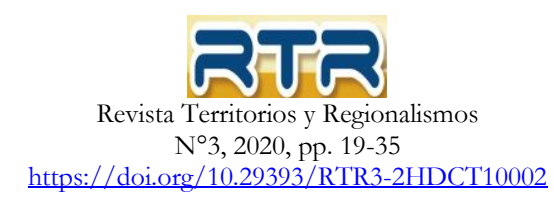

4) Personas (Smart People): Un factor diferenciador de una smart city, es que cuenta con una ciudadanía inteligente. En una ciudad inteligente cuya comunidad ha "aprendido a aprender"; los cambios en la ciudad son promovidos por sus habitantes quienes, contando con la formación y el apoyo técnico adecuado, emprender acciones que previenen el consumo excesivo de energía y la contaminación del medio ambiente. Lo que genera una retroalimentación al sistema que potencia la calidad de vida de la ciudad, haciéndola más competitiva y sostenible.

5) Calidad de vida (Smart living): La ciudad smart es más eficiente y ofrece desarrollo económico, lo que lleva a ofrecer mejores servicios y a su vez mejora la calidad de vida de la población. Es atractiva para los ciudadanos, empresarios y trabajadores que quieren tener éxito, promueve el desarrollo de soluciones creativas, genera empleos y disminuye la desigualdad. De esta forma termina creando un círculo virtuoso que produce bienestar económico y social (BID, 2016).

6) Gestión y administración inteligente (Smart governance): En la gestión smart, los procesos de la administración pública y finanzas son digitalizados, generando un acceso ágil a los datos públicos. Promoviendo la creación y uso de servicios públicos en línea, la e-Democracia, el Gobierno transparente (Azkuna, 2012); permitiendo a su vez que los funcionarios públicos, de diferentes departamentos, trabajen como equipo en una visión colaborativa, integrada y siempre actualizada (BID, 2016). Evitando así la crítica más frecuente hacia la gestión pública, "que cada unidad, división o subsecretaría trabaja como una isla separada, y en el peor de los casos, con franco antagonismo, mala comunicación y traslapo de funciones entre ellas" (Waissbluth, 2003) .

Luego de revisar en detalles las distintas definiciones sobre smart city y las dimensiones que componen una ciudad inteligente se cree importante hacer un contraste de la ciudad de Concepción bajo las condiciones presentadas y verificar si esta cumple o no con ellos.

Para este contraste es útil el "Ranking de Ciudades inteligentes en Chile" (Cohen \& Obediente, 2014), el cual utiliza 28 indicadores que agrupa en seis ejes, las dimensiones que componen una smart city presentadas en el apartado anterior, los cuales no pretenden establecer "un modelo único", sino, presentar las bases de un modelo que se retroalimente en función de las necesidades locales que se vayan identificando y las nuevas oportunidades que se generan a raíz de estos.

El fundamento de este contraste, el cual es compartido con el ranking mencionado, fue tener una base o "hoja de ruta", para identificar dónde se posicionaba la ciudad de Concepción y hacia donde debe apuntar con el objetivo de llegar a ser una ciudad inteligente.

Los indicadores del ranking fueron desarrollados conforme diversas bases de datos públicos, de diferentes organismos gubernamentales y no - gubernamentales, involucrados en la información requerida por medio de solicitud de esta a través del Portal de Transparencia Activa ${ }^{4}$ de las respectivas municipalidades que fueron parte del estudio.

Luego de recopilar toda la información solicitada se generó la base de datos de los 28 indicadores, asignándole a cada uno el mismo peso específico con el fin de presentar resultado objetivos. Bajo esa medición ${ }^{5}$ la ciudad de Concepción fue clasificada quinta a nivel nacional:

\begin{tabular}{|l|l|l|l|l|l|l|l|l|}
\hline Ranking & Ciudad & $\begin{array}{l}\text { Medio } \\
\text { ambiente }\end{array}$ & Movilidad & Gobierno & Economía & Sociedad & $\begin{array}{l}\text { Calidad } \\
\text { de vida }\end{array}$ & \begin{tabular}{c} 
Total \\
\hline 5
\end{tabular} \\
\hline
\end{tabular}

Fuente de elaboración propia en base a (Cohen \& Obediente, 2014)

\footnotetext{
${ }^{4}$ Ley 20.285, Sobre Acceso a la Información Pública.

5 La información fue procesada por tres métodos: 1) Promedio ponderado para agrupar las ciudades con más de una comuna. 2) Z-score para normalizar cada indicador. 3) Transformación de los resultados generales de Z-Score en una escala de base 15 puntos. (Cohen \& Obediente, 2014)
} 


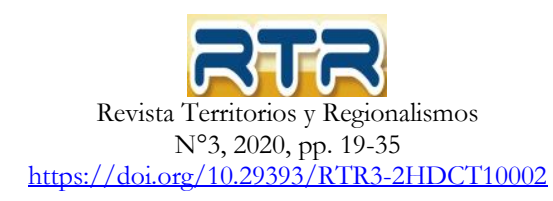

En un resumen de cada ciudad se señala sobre Concepción que:

Los ejes de mayor valor son los de sociedad y gobierno, los que incluso superan a la media nacional en base al estudio. Sin embargo, las dimensiones de economía y calidad de vida se quedan atrás en los parámetros establecidos, siendo bajos y el eje de economía en particular el más bajo a nivel comunal.

En relación de medio ambiente era alarmante el nivel de $\mathrm{CO} 2$ per cápita, para el entonces era de un 16,04 Ton CO2 por año, siendo el más alto entre las ciudades evaluadas, que tienen un promedio 4.40 CO2 al año.

En el eje de movilidad los indicadores eran positivos, en relación con otras ciudades evaluadas. Lo anterior se funda en que la comuna cuenta con el sistema de Biotrén, que funciona con electricidad, por lo tanto, no genera emisiones de CO2. Destacando además que, en el año en que se realizó la medición la ciudad había comenzado el proceso de construcción de ciclovía, con un total de siete distribuidas en cerca de 10 kilómetros de vía, impulsando un sistema de movilidad sin emisiones.

Se destaca de forma positiva a la ciudad como líder en el indicador de graduados universitarios, por cada 1.000 habitantes, alcanzando la tasa más alta, con 55 graduados por cada 1.000 habitantes versus 27.09 graduados, que corresponde al valor promedio de las ciudades evaluadas.

A su vez, los indicadores que se ubicaron muy por debajo del promedio fue la tasa de criminalidad y la asignación presupuestaria en cultura, que era la más baja para el momento de la medición, $0,5 \%$ versus $1,87 \%$ promedio.

Para concluir este apartado es menester señalar que la implementación de este nuevo modelo de desarrollo de ciudad requiere la creación de un adecuado sistema de gestión y administración, aprovechando las tecnologías más innovadoras en función de esta. Dicho aspecto incluye generar planes estratégicos para la promoción de las TICs en función de la administración local (Azkuna, 2012).

Se define entonces smart governance, como un gobierno a la cabeza de una región o smart city que, desde el punto de vista del ciudadano, lo considera cercano a él, previsible y transparente en la toma de decisiones, y que cuenta con ellos. También, dicho gobierno tiene que abogar por aplicar políticas sociales y medioambientales, y hacer uso de la tecnología para conseguir una interconexión más directa entre la autoridad y el ciudadano (López, 2013).

Concluyendo este apartado se agrega el trabajo del académico norteamericano Dr. Boyd Cohen, quién describió la implementación de ciudades inteligentes como un proceso de 3 fases (Cohen, 2015):

Smart Cities 1.0: Es la etapa donde proyectos ofrecidos por proveedores de tecnologías a municipios son desechados por estos últimos al no comprender las oportunidades de solución que la tecnología puede ofrecer a mejorar la calidad de vida de los ciudadanos.

Smart Cities 2.0: Ahora son los municipios quienes entienden el potencial de la tecnología en la función pública, y son ellos quienes definen proyectos con miras en la tecnología que permita mejorar la calidad de vida en la ciudad. Esta es la fase por la cual ciudades como Málaga, Concepción y Santiago están cursando, en donde son los alcaldes quienes desean que sus ciudades sean más smart y para ello solicitan ayuda (apoyo técnico - teórico, financiamiento, respaldo social, entre otros) para iniciar esta transformación.

Smart Cities 3.0: Se distingue un elemento diferenciador en donde son los ciudadanos quienes, en una participación en el proyecto, colaboran en diseñar la próxima generación de ciudades inteligentes, y más sostenibles.

Una ciudad inteligente será una ciudad cuya comunidad ha "aprendido a aprender", adaptarse e innovar (Coe et al., 2001), de lo que se deduce la necesidad de adaptación por parte de la población, quienes deben ser capaces de utilizar la tecnología con el fin de beneficiarse de ella. 


\section{El "triángulo smart" forma y estructura de la smart city: Capital social, alianza público-privada y Gobierno orientado a la innovación.}

\section{Capital Social}

Una ciudad que aspira a ser considerada smart, debe necesariamente incorporar aspectos relativos a la mejora de la gobernanza, la planificación y de la infraestructura, de tal manera que estas se reflejen en su capital humano y social.

La smart city es aquella que posiciona a las personas como centro de su desarrollo, incorpora TICs en la gestión urbana, y usa las herramientas que le entrega para estimular la formación de un gobierno eficiente que incluye procesos de planificación colaborativa y participación ciudadana.

La transformación y modernización de la gestión en la ciudad genera una serie de resultados concretos y positivos, la smart city se torna más innovadora, competitiva, atractiva y resiliente, aumentando la eficiencia de la administración local al integrar diferentes áreas de actuación. Trabajando en forma colaborativa, los administradores municipales comparten información de calidad para ofrecer mejores servicios a la población (BID, 2016).

En relación con lo anterior, es necesario comprender que es una sociedad del conocimiento. Se considera una sociedad del conocimiento aquellas que cuentan con una capacidad para generar conocimiento sobre su realidad y su entorno, y cuenta con la capacidad para utilizar dicho conocimiento en el proceso de construir su futuro (Chaparro, 2001, p. 28). Y es en base a este conocimiento socializado, o capital social, que la administración pública puede dar respuesta a los desafíos y oportunidades que este nuevo contexto social le brinda; como la obligación de contar con la capacidad de adaptarse e integrar creativamente ese entorno, al mismo tiempo que crea nuevas realidades relacionadas con sus metas y objetivos.

Lo que es propuesto en este trabajo de investigación es que la ciudad de Concepción migre de un modelo tradicional de gestión a uno de smart city. Pero lo anterior no se logrará si no se desarrolla en ella una conciencia cívica por parte de los ciudadanos, ya que para sustentar este proyecto es necesario una red de apoyo, relacionando a la administración pública con la comunidad, donde los intereses comunes puedan lograrse a partir de esfuerzos colectivos y así obtener beneficios mutuos.

El fomento de acciones de beneficio colectivo, la interacción y redes sociales, la confianza, así como el sentido de pertenencia y responsabilidad hacen posible el mejor intercambio de recursos y la colaboración entre los distintos actores sociales, en sus diferentes formas como son: individual, organizacional y externo.

Lo anterior se relaciona con enfoques tanto culturales como estructurales, cuando se estudia el concepto de capital social.

Los académicos especializados en el tema suelen considerarlo como el conjunto de normas, redes y organizaciones construidas sobre relaciones de confianza, que contribuyen a la cohesión, el bienestar y el desarrollo de la sociedad, así como la capacidad de los miembros de esta para actuar en forma coordinada y así satisfacer sus necesidades en función de un beneficio general.

Fue el sociólogo francés Pierre Bourdieu, en el año 1980, quien dio una definición del capital social definiéndolo como el conjunto de recursos presentes o potenciales que son vinculados a la existencia de una red sostenible de relaciones más o menos institucionalizadas de inter-conocimiento y de interreconocimiento; o, en otros términos, a la pertenencia de un grupo, como conjunto de agentes que no están únicamente dotados de propiedades comunes si no que están unidos por enlaces permanentes y útiles (Bourdieu, 1980).

Chaparro (2001), Kliksberg (2006) y Medina González et al., (2015) complementan la idea de aquel concepto/factor "invisible" que emerge desde las relaciones cotidianas, habituales de los miembros de una comunidad, robusteciendo sus vínculos, haciendo que estos compartan su conocimientos en beneficio del otro, con el fin de un beneficio mutuo y apropiándose de este conocimiento generado así 


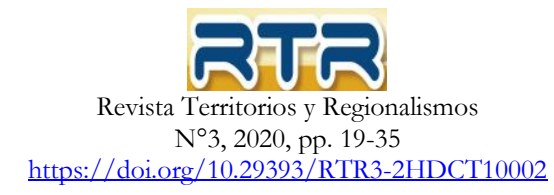

el factor que en la economía actual recae mayor importancia, como lo es el conocimiento socializado o capital social.

\section{Alianza público-privada (APP)}

Como consecuencia de la modernización, la búsqueda del desarrollo nacional y de la creciente complejidad de la sociedad actual, las instituciones públicas están pasando de ser proveedoras directas de bienes y servicios públicos a reguladores y contratistas de dichos servicios (Allan, 2001).

Esto ha derivado en un aumento exponencial en las Alianzas Público-Privada (APP) en las últimas décadas, para llevar acabo la construcción de carreteras, puentes, aeropuertos, metros o puertos marítimos; ofrecer servicios de abastecimiento de agua, electricidad, gas o acceso a telecomunicaciones; crear y gestionar sistemas judiciales, prisiones, hospitales, colegios centros deportivos; y hasta mejorar la calidad y acceso a viviendas sociales y servicios básicos (Casado, 2007).

Devlin \& Moguillansky (2009) menciona que son pocos los países en desarrollo que han logrado disminuir sostenidamente las brechas de ingresos con los países desarrollados, sin una acción proactiva del gobierno en colaboración con el mercado; lo cual les ha permitido avanzar en una transformación productiva con participación e inserción dinámica en el ámbito internacional.

Tabla conceptual: Requisitos importantes para que las APPs sean un instrumento de las estrategias de desarrollo.

\begin{tabular}{|l|l|}
\hline $\begin{array}{l}\text { Requisito } \\
1\end{array}$ & $\begin{array}{l}\text { Colaboración del Estado y el sector privado, pero resguardando el marco público. Debe } \\
\text { evitar ser "capturado" por intereses particulares. }\end{array}$ \\
\hline $\begin{array}{l}\text { Requisito } \\
2\end{array}$ & $\begin{array}{l}\text { Diseño institucional colaborativo público-privado, que permita identificar las nuevas } \\
\text { oportunidades y resolver dificultades productivas. }\end{array}$ \\
\hline $\begin{array}{l}\text { Requisito } \\
3\end{array}$ & $\begin{array}{l}\text { Generar raíces profundas que perduren en el tiempo, generando consenso y aceptación por } \\
\text { parte de la opinión pública. }\end{array}$ \\
\hline
\end{tabular}

Fuente: Elaboración propia en base a "Alianzas público-privada como estrategias nacionales de desarrollo a largo plazo" (Devlin \& Moguillansky, 2009).

\section{Gobierno orientado a la innovación. (Smart Governance)}

Bien utilizadas, las TICs no quedan sólo en excelentes herramientas en función de la administración pública. Esta es la característica principal de un gobierno smart, ya que este entiende que las tecnologías están en función de facilitar los procesos de apropiación social de lo público y a su vez en la creación de lo público.

Teniendo lo anterior en consideración hoy en día, el desarrollo y las tecnologías avanzadas resultan imprescindibles para la administración de las ciudades (Sikora - Fernández, 2017), los campos en los que puede actuar un gobierno asociado a las TICs para una buena gestión son ilimitados.

Este aspecto incluye una administración pública inteligente, capaz de crear conocimiento y darle uso en la práctica (Giffinger et al., 2007). Dicho aspecto incluye la promoción de planes estratégicos para la promoción de las TICs en función de la administración local (Azkuna, 2012).

Como ejemplo de lo anterior, la municipalidad de Concepción presenta su "MuniConceApp"6, inaugurando una herramienta tecnológica en función de su proyecto de cambio a una gestión smart.

Sin embargo, el uso de las TICs debe ser entendida como lo que es, un medio que facilita y genera las condiciones para emprender ese cambio y no un fin en sí mismo (BID, 2016).

${ }^{6}$ Aplicación móvil que permite a los habitantes de la comuna a realizar trámites online. A lo cual se agregan puntos de conexión Wifi gratis en puntos específicos de la ciudad, transformándose así estos en herramientas y un vínculo entre los ciudadanos y la gestión local. 


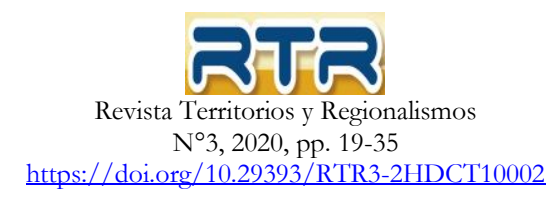

Por vía de estas y otros medios utilizados, se intenta mejorar la toma de decisiones, realizar una administración eficiente, suministrar la prestación de los servicios producidos y potenciar la competitividad. Algunos definen smart governance, como un gobierno a la cabeza de una región o smart city que, desde el punto de vista del ciudadano, lo considera cercano a él, previsible y transparente en la toma de decisiones, y que cuenta con ellos.

También, dicho gobierno tiene que abogar por aplicar políticas sociales y medioambientales, y hacer uso de la tecnología para conseguir una interconexión más directa entre la autoridad y el ciudadano (López, 2013).

Triangulo Smart: Condiciones y estructura de una ciudad inteligente

Para concluir esta sección, en base a la investigación de Jorge Sábato \& Natalio Botana, titulado "La ciencia y la tecnología en el desarrollo futuro de América Latina" (1993), que a pesar del año de su creación su contenido sigue influyendo investigaciones actuales en relación al tema central de su obra, especialmente por su "triángulo de relación entre el gobierno, ciencia-tecnología y estructura productiva" que se presenta a continuación.

Un gobierno smart, en alianza público-privada de mutua colaboración y beneficio para el sector público y privado, tiene una gran e importante tarea, insertar a la ciencia y la tecnología en el proceso mismo del desarrollo, lo cual le impone el desafío de saber el dónde y cómo innovar. Con el objetivo de superar las condiciones desfavorables a través del desarrollo de un sistema productivo actualizado a las demandas y exigencias de los tiempos actuales.

Las experiencias, históricas como contemporáneas, demuestran que este proceso político es el resultado de la acción múltiple y coordinada de tres actores protagonistas en el desarrollo de las sociedades actuales; en donde se establece un sistema de relaciones e interacciones entre ellos, que se representa por un triángulo donde cada uno ocuparía un vértice de la figura (Sábato \& Botana, 1993).

A continuación, se presentan y definen los conceptos que lo componen:

El Gobierno: Conjunto de instituciones que tienen como objetivo formular políticas y movilizar recursos de y hacia los vértices de la estructura productiva y de la infraestructura científico - tecnológico, por medio de procesos legislativos y administrativos.

La estructura productiva: Conjunto de sectores productivos que proveen de bienes y servicios que demanda la sociedad y surge desde ella.

La infraestructura cientifico-tecnológica: Aquella que es compuesta por: el sistema educativo, laboratorios, sistema institucional de planificación y promoción de la investigación, mecanismos jurídicoadministrativo que regula el funcionamiento de las instituciones y los recursos económicos y financieros aplicados a su funcionamiento.

Triángulo Smart de relaciones entre gobierno, ciencia - tecnología y estructura productiva. Gobierno (Gobierno orientado a la innovación)

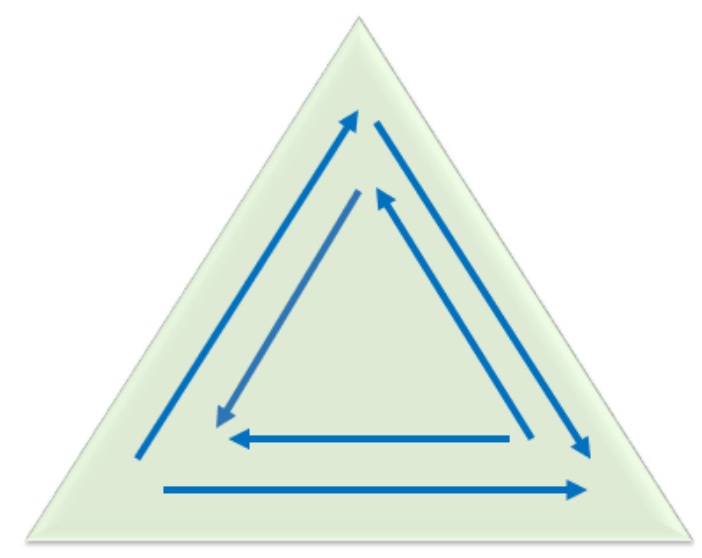

Estructura productiva Infraestructura científico- tecnológico (Capital social) (Alianza público-privado)

Fuente de elaboración propia en base a (Sábato \& Botana, 1993) 


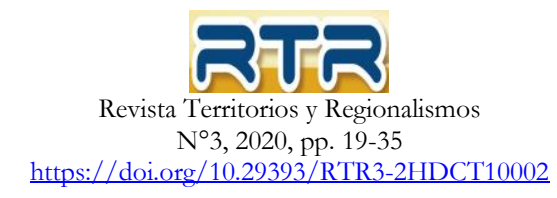

En síntesis, la smart city es una ciudad que responde de forma rápida a las necesidades presentadas por su población, a la vez que mejora la calidad de vida de sus de sus habitantes.

Esta capacidad es generada por un crecimiento sostenible de la ciudad; innovación social; APP entre centros de investigación, universidades, socios privados y públicos en el ámbito de proyectos; y una gestión inteligente que ofrece sus servicios a la comunidad de forma flexible, continua y ágil a través de las TICs (BID, 2016).

Para concluir se enfatiza que, el gobierno, y su programa de construcción de smart cities, debe adquirir una actitud proactiva, donde la colaboración público-privada proporcione las condiciones necesarias para que la innovación por medio de las TICs fomente la implementación eficaz de un programa estratégico de desarrollo, en función de promover la productividad a mediano y largo plazo, orientada a la inserción internacional.

\section{Representantes de la ciudad de Concepción: experiencia, opiniones y propuestas.}

Para la realización de esta investigación se desarrollaron entrevistas semi-estructuradas a personas relacionadas y/o representante de cada una de las condiciones de la implementación de una smart city en la ciudad de Concepción, las cuales son: capital social, alianzas público - privada y un gobierno orientado a la innovación.

Los hallazgos, fruto de estas entrevistas, fueron categorizadas en cada una de las condiciones de esta investigación, con el fin de esclarecer cual es la postura y/o percepción de cada uno de los/as entrevistados con las condiciones actuales de la ciudad de Concepción a miras de ser una smart city futura. Considerando sus posibilidades y desafíos.

\section{Una agenda incipiente}

Desde el comienzo de esta recopilación de información por medio de entrevistas - semi estructuradas, se presenció un panorama de opiniones contrapuestas. Ya que, en relación con la primera pregunta, tenemos percepciones favorables como la del director regional del Instituto de la Juventud (INJUV), el señor Gustavo Apablaza quien dice que "en los últimos años, sí. Sobre todo, en las ciudades más grandes como Concepción, si se ha puesto en la palestra hablar de la smart city sobre todo vista desde los gobiernos comunales y se ha estado conversando" (G, Apablaza, 2019).

En la misma línea, el señor Pedro Venegas, Director de Planificación comunal, de la Ilustre Municipalidad de Concepción, quien en sintonía con el director regional del INJUV menciona que en su opinión "hace unos 5 o 6 años viene siendo un elemento de análisis tanto para el sector público como para el sector privado. La forma de desarrollar a nivel local, lo que puede ser la iniciativa de crear en Concepción o en otras ciudades de la región este mecanismo de ser una ciudad inteligente" (P, Venegas, 2019).

Desde una mirada no tan "optimista", el Director de Desarrollo e Innovación de la Universidad de Concepción, el señor Claudio Maggi, menciona que desde su percepción sobre si la smart city ha sido un tema de debate en el ámbito público - privado en Concepción, menciona que en su opinión ha sido "Poco. Yo te diría que esto es un tema incipiente. No está muy instalado, está más instalado como política pública" (C, Maggi, 2019). Además, desde su rol de director de desarrollo de la Universidad de Concepción, menciona que "en la academia todavía, en particular, en el núcleo de la universidad recién se está entendiendo como se puede expresar, el concepto de ciudad inteligente en nuestro entorno urbano en este caso en la ciudad de Concepción" (C, Maggi, 2019).

Con una opinión más categórica, la Directora Regional de Corfo BioBio, Sra. Macarena Vera Messar, señala que su opinión en relación con la pregunta en cuestión indica que "no. Yo creo que estamos aún al debe en eso, ha habido debate, público-privado si acá en la región, pero falta aún que sea un tema más relevante" (M, Vera Messar, 2019). Comenta además que en su opinión “estamos en pañales 


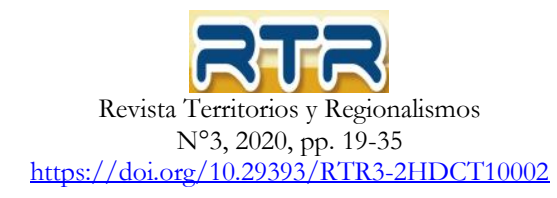

por decirlo así, a pesar de que como Corfo Biobío hemos estado desarrollando debates y encuentros, falta aún más" (M, Vera Messar, 2019).

Se logra apreciar una línea argumentativa similar entre los entrevistados, en este apartado de la pregunta, donde se considera que las "características de las innovaciones tecnológicas en especial las digitales, cuando se incorporan como innovación, el proceso de maduración es lento, lento, lento, sin embargo, hay un instante donde surge una ruptura" (J, Durán, 2019), la cual permitirá la "apropiación social" (Chaparro, 2001) del nuevo modelo de ciudad.

En relación con los incentivos se desmarcan las posturas más antagónicas. Sobre la existencia de incentivos la directora regional de Corfo BioBio señala que "Si, Claro. El comité de desarrollo productivo tiene incentivos para ello, por eso es por lo que hemos desarrollado mesas de trabajo, encuentros con más de 150 actores relevantes, se han identificado las brechas, más que brechas, cuáles son las principales temáticas que son necesarias trabajar sobre smart city acá en la región” (M, Vera Messar, 2019).

$\mathrm{Y}$, en respuesta a la misma pregunta el Director de Planificación municipal menciona que "el Estado chileno hoy en día no establece ningún incentivo al respecto. La idea de poder motivar a las distintas comunas para que puedan definir una estrategia en ese ámbito, pero con recursos propios" ( $\mathrm{P}$, Venegas, 2019).

De lo cual se desprende, que no es por falta de incentivos, sino de comunicación que hay entre los órganos del Estado lo que genera impedimentos para realizar proyectos, como el propuesto en este trabajo de investigación, demostrando así una vez más que la "insularidad" (Waissbluth, 2003) de los agentes públicos generan problemas y los ciudadanos sufren las consecuencias.

Construir una estrategia en cooperación público-privada

Autores consultados y representantes comunales entrevistados coinciden en que una ciudad inteligente es aquella que "pone" al ciudadano en el centro de su actuar, y utiliza las nuevas herramientas tecnológicas, TICs, Big Data, democracia digital, etc., con el fin de mejorar las condiciones de vida de todos sus habitantes.

Por lo cual, "antes de transformarla en una smart city, lo que nosotros tenemos que preocuparnos, es trasformar a Concepción en una ciudad a escala humana” (P, Venegas, 2019).

Quien mejor que el Director de Planificación comunal nos indique que esta primera fase de implementación de ciudad inteligente en Concepción sea entendida como aquella "que está orientada a establecer una relación más horizontal con la comunidad y en esa lógica a lo mejor la relación con la smart city tiene que ver con requerimientos que la propia comunidad pueda, en su momento, ir levantando lo necesario para poder mantenerse interconectada" (P, Venegas, 2019).

Una segunda fase seria "incorporar, la idea de ciudad inteligente, dentro de las temáticas regionales, incorporarla dentro de los planes regionales de los servicios públicos, por ejemplo: Que INJUV hable de ciudades inteligentes, que SERCOTEC hable de smart city” (M, Vera Messar, 2019), al igual que CORFO, etc.

Terminando así con una tercera fase de colaboración, "lo cual es esencial entre el sector público, la sociedad civil y el sector privado para poder apuntar a soluciones tripartita” (G, Apablaza, 2019).

Por un lado, se es enfático al mencionar que “iTotalmente! Totalmente, y no solo público-privada, yo le colocaría ahí, público, privada y la academia. Tiene que haber un triángulo donde conversan público, privado y la academia, es el triángulo perfecto para hablar de cualquier iniciativa" (M, Vera Messar, 2019).

Y respondiendo toda duda, en caso de que hubiera, se menciona por otros entrevistados en relación con promover las alianzas estratégicas "que no lo puedes no hacer, ósea, una smart city requiere alianzas público privada" (C, Maggi, 2019) y es así por qué se considera "un elemento fundamental para el desarrollo de la ciudad. Es indispensable para que avance o se desarrolle la ciudad contar con esa alianza estratégica, es clave" (G, Apablaza, 2019).

En una propuesta complementaria y en línea con la base de esta investigación que es la literatura especializada y el plan nacional, el director de desarrollo e innovación, menciona que "lo clave que hizo 


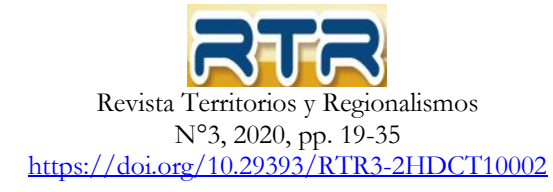

Santiago, es crear un núcleo de acción de cuatro hélices: 1) ámbito privado, 2) ámbito público, 3) academia y 4) sociedad civil" (C, Maggi, 2019).

Primero identificando las "brechas de la ciudad de Concepción actual con una smart city futura", para luego dar paso a esa hélice de trabajo con cuatro puntas y promocionar estas, y el trabajo que realizan, por medio de "conferencias de ciudades inteligentes en Chile" (J, Durán, 2019).

\section{Visualizando la agenda de un Concepción Inteligente}

Así como en la primera pregunta, en esta se mantiene una línea argumentativa similar ya que se coincide en que "primero (se debe) definir cuáles son las necesidades que la ciudadanía cree que son más relevantes y luego empezar a desarrollar temáticas de trabajo en cada una de ellas" (M, Vera Messar, 2019).

Derivado de esa consulta y/o levantamiento de información se categorizaría en rango de prioridad para la ciudadanía, debido a cuáles son las alianzas publico privado que más se debería trabajar; se menciona por parte de los entrevistados que hay ámbitos de prioridad y el director Claudio Maggi, señala que a su parecer, es preciso mencionar que estos se pueden agrupar en: 1) Movilidad, 2) Seguridad, 3) Eficiencia energética y 4) Experiencia ciudadana (C, Maggi, 2019).

Al momento de consultar por la existencia de estas en la practica el ánimo en las respuestas cae y ya no son tan favorables. Salvo por el Director de Planificación comunal quien indica que "se están desarrollando en transporte, conectividad, accesibilidad y sistemas de cámaras de vigilancia. Lo que se está desarrollando con instituciones públicas, como lo es en este caso la subsecretaria de prevención del delito y con carabineros de Chile y una empresa privada que le hace mantención al sistema" (P, Venegas, 2019).

Lo cual discrepa de lo que mencionan otros entrevistados como el director regional del INJUV BioBio quién señala que a su entender "qué yo conozca, no", en relación con la existencia de las alianzas público - privado en la práctica (G, Apablaza, 2019).

Queda en evidencia una vez más la falta de comunicación entre un organismo y otro, obstaculizando así la posibilidad de nuevas formas y/o herramientas que posibiliten entregar un mejor servicio a los ciudadanos.

\section{El capital social existente y educación pertinente}

Para dar una solución y/o herramienta útil para mitigar aquella falta de participación y la no existencia de este movimiento social que potenciaría aún más la idea de implementar una ciudad inteligente en la capital de la región del BioBio, "propondría hacer un artículo simple, no complejo, que muestre a la ciudadanía cuáles pueden ser los beneficios de una ciudad inteligente, pensando que tenemos tecnologías para modernizar fuertemente muchos servicios" (J, Durán, 2019), siendo estos para el beneficio de la ciudadanía y una mejor gestión territorial.

Es compartida la percepción de "que la comunidad hoy está con interés de participar". La pregunta es, “¿en qué quiere participar?" (P, Venegas, 2019). Eh ahí que surge una de las primeras propuestas en relación con la pregunta en cuestión, este entrevistado menciona que "propondría que se instale un observatorio de participación en relación con el desempeño de la ciudad en atributos de una smart city permitiendo obtener indicadores que regularmente se estén consultando y que además frente a una propuesta de ciudad inteligente, haya también una opinión ciudadana que se recoja" (C, Maggi, 2019).

En relación ahora, con las vías de llevar acabo la participación ciudadana se indica que "hay tecnología, hay now how, probablemente hay plata, hay que ver que podemos hacer" ... "Las herramientas están disponibles y no las usamos" (J, Durán, 2019). Como se aprecia hay condiciones para promover la idea fundacional de esta investigación, pero no es suficiente con que existan, estas también deben ser llevadas a la práctica. Y ante ello. La directora de CORFO BioBio es clara en señalar un camino a seguir, 


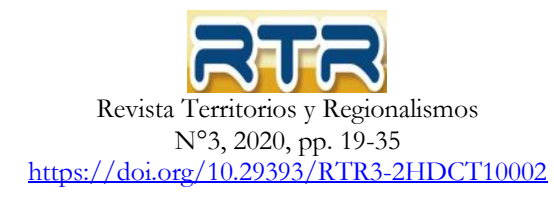

"primero, hacer entender a la ciudadanía el concepto de smart city; segundo, involucrar a nuestras autoridades y tercero, hacer alianzas y conversaciones de mesas, entre lo público, privado y la academia. Hay que hablar más de smart city" (M, Vera Messar, 2019).

Por parte del jefe de la unidad de educación a distancia de la Universidad de Concepción, José Durán, menciona que "mi misión como profesor, como ingeniero, como persona, ha muy temprano fue extender la educación hacia sitios que se necesitan, es decir, no fronteras para la educación" (J, Durán, 2019).

Y el director de desarrollo e innovación de la misma casa de estudios, menciona que "la eeducación no es un tema que uno pueda encontrar bueno o malo, es ".." me guste o no me guste, viene. Todas las entidades educativas nos tenemos que aprontar a que una parte importante de nuestra interfaz va a ser virtual, no es casualidad que el segundo proyecto del bicentenario sea el "campus digital"; me parece, que es como si me preguntaras, ¿Qué me parece el internet?, me parece que sí, la e-educación viene, y la pregunta no es si nos subimos o no, la pregunta es cómo nos subimos y no nos quedamos atrás" (C, Maggi, 2019).

Es importante y necesario para proyectos que tienen como fin el desarrollo de la ciudad de Concepción o cualquier otra ciudad del país, cuando vemos una realidad en la que condiciones tan importante para ellos se nos retrata una realidad en donde se atestigua que "ahí sí que estamos débil". "Yo creo que se están creando profesionales que la industria a veces no necesita, la industria, hoy en día... salen 100 ingenieros, pero ¿Qué busca la industria en un ingeniero?, hoy en día deberíamos tener carreras más abiertas, con un enfoque más técnico, más de oficio, en el fondo, falta especificación de lo que estrega la universidad” (M, Vera Messar, 2019).

En línea con el micro retrato de la realidad en relación a la pregunta, se plantea que "es clave para el desarrollo que la universidad se vincule con la empresa, para el ecosistema de innovación, si tú haces solo investigación y no aplicas queda cojo el sistema, ósea, si nosotros queremos transformarnos en una smart city y queremos tener fuentes productivas más integradas, la universidad necesariamente tiene que vincularse con el sector productivo", sentencia el director regional del INJUV, finalizando su argumento con una opinión con tinte propositivo en la cual señala que "ahí estamos al debe, y de los dos lados, porque las universidades quieren vincularse, pero les falta un poco dar el paso" (G, Apablaza, 2019).

\section{Lograr un Gobierno orientado a la innovación.}

Se comenta que "estamos bajos todavía en infraestructura tecnología, somos una región, una ciudad con una tremenda oportunidad de hacer cosas, tenemos un mundo universitario tremendo, pero baja infraestructura y eso implica una fuga de talentos" (M, Vera Messar, 2019).

Con un "diagnóstico" a nivel regional resulta útil también saber cómo es el panorama nacional bajo la pregunta en cuestión y se nos menciona que "hace años que el país ha venido trabajando en una agenda digital que tiene distintos componentes, pero uno de esos siempre ha sido el gobierno digital, para facilitar trámites, eliminar papeleo, unificar acceso clave y eso todavía está lejos de producirse" (C, Maggi, 2019).

Desde la administración pública comunal se ha adquirido la noción que se "tiene dos visiones" sobre la infraestructura tecnológica, la cual depende del enfoque en su implementación.

Si se presenta un mal uso de la información solicitada para un análisis de "quienes son las personas que actúan y los extranjeros que participan en la crisis social", para ello se utiliza la tecnología y análisis del Big Data para crear información falsa, los gobiernos de ese "determinado Estado", van en la dirección incorrecta". Sentencia el director de planificación comunal, continuando con la otra cara de su análisis, comenta que "la línea correcta, de las TICs es el uso de las nuevas tecnologías de información para poder potenciar o focalizar programas sociales, por ejemplo, para poder impulsar el desarrollo productivo de determinada área, esa información, esos tipos de mecanismos si son más útiles” (P, Venegas, 2019). 


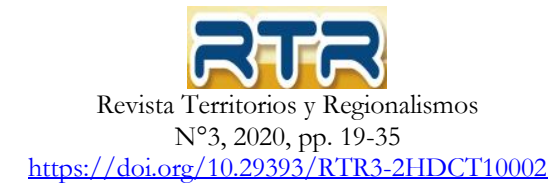

Las TICs tienen un rol fundamental, para que la ciudad de Concepción migre a una de smart city, eso está claro, pero el rol que cumplen no es el protagónico. Ese está reservado para otros actores, para aquellos ciudadanos "smart" que se "apropien de lo público" y hagan de esta idea una realidad.

"Estas TICs de las que tú hablas deben ser incorporadas a los servicios públicos, nosotros acá no podemos seguir liderando servicios públicos con una administración pública retrógrada sin uso de las TICs, es decir sin herramientas tecnológicas. Ósea, no puede ser, que todavía exista el papelito, que la cosa... hoy en día todos nuestros instrumentos son a través de una plataforma, pero todavía se nos cae la plataforma, todavía no hay herramientas tecnológicas que permitan llegar a esta smart city, entonces hay que parar evidentemente en los servicios públicos y las instituciones tienen que moverse en ese sentido" (M, Vera Messar, 2019).

\section{Conclusiones: Camino a seguir, propuesta y reflexiones finales sobre Concepción, Ciudad Inteligente.}

Lo que se propone en este trabajo de investigación, en base a la literatura y la experiencia y opiniones de informantes clave, es que la ciudad de Concepción migre de un modelo tradicional de gestión a uno de smart city.

Además de derribar mitos sobre la inexistencia de incentivos para proyectos de ciudades inteligentes a nivel comunal. Lo cual, se demuestra en que "el comité de desarrollo productivo tiene incentivos y es por ello que se han desarrollado mesas de trabajo con más de 150 actores relevantes, se identificaron las brechas, pero más que brechas, se identificaron cuáles son las principales temáticas necesarias a trabajar sobre una smart city en la región” (M, Vera Messar, 2019).

Pero lo anterior no se logrará, si no se desarrolla en la ciudad, una conciencia cívica por parte de los ciudadanos, ya que, para sustentar este proyecto es necesaria una red de apoyo, relacionando a la administración pública con la comunidad, donde los intereses comunes puedan lograrse a partir de esfuerzos colectivos y así obtener beneficios mutuos.

Con forme lo anterior, la directora de CORFO BioBio es clara en señalar un camino a seguir, "primero, hacer entender a la ciudadanía el concepto de smart city; segundo, involucrar a nuestras autoridades y tercero, hacer alianzas y conversaciones de mesas, entre lo público, privado y la academia. Hay que hablar más de smart city" (M, Vera Messar, 2019).

El foco debe estar en este "camino a seguir" de trabajo transversal y colaborativo, donde representantes del mundo privado, público y la academia, tengan como objetivo y visión común ver a la ciudad de Concepción más innovadora e inteligente, trabajen y realicen acciones y planes de trabajo colaborativo con el fin de realizar y cumplir el objetivo que los une. Para lo cual, se presentó un antecedente necesario, el "Ranking de Ciudades Inteligentes en Chile", elaborado por el experto en estrategia urbana Boyd Cohen y Elizabeth Obediente, (Cohen \& Obediente, 2014). Según el ranking de Cohen y Obediente (2014), en una selección de 28 indicadores Concepción era la quinta ciudad del país.

Los ejes de mayor valor incluso superaban la media nacional, los cuales son sociedad y gobierno. Donde economía y calidad de vida se quedaban atrás en los parámetros establecidos, siendo bajos y el indicador de economía en particular el más bajo a nivel comunal.

En consideración de que "un proyecto transformador de ciudad inteligente comienza con un estudio detallado de problemas considerados prioritarios y que afectan al mayor número de personas" (BID, 2016, p. 49).

A título de este investigador se propone realizar nuevamente este análisis comunal, en base a estos indicadores de gestión, para verificar cuál es el estado actual de la ciudad y asimilarlo a ser el "antecedente base" de futuras mediciones. 


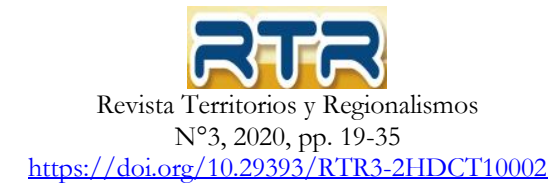

Además, "propondría que se instale un observatorio de participación en relación con el desempeño de la ciudad en atributos de una smart city y que con eso tenga indicadores que regularmente se estén consultando y que además frente a una propuesta de ciudad inteligente, haya también una opinión ciudadana que se recoja" (C, Maggi, 2019).

Para lo cual "es clave que la universidad se vincule con la empresa, para crear un ecosistema de innovación, si nosotros queremos transformarnos en una smart city y queremos tener fuentes productivas más integradas, la universidad necesariamente tiene que vincularse con el sector productivo" (G, Apablaza, 2019); lo anterior directamente vinculado con el observatorio aquí propuesto.

Para concluir, se apuesta por un trabajo transversal por la ciudad. Trabajar de forma colaborativa con todas aquellas organizaciones, privadas, públicas y la academia, que quieran colaborar y de esta forma ayudar a que la ciudad de Concepción migre de una administración tradicional a una de ciudad inteligente, velando por priorizar las áreas más deficientes de la ciudad y que posiblemente estén afectando más la calidad de vida de sus ciudadanos.

Clarificando, que no es por falta de incentivos, sino de comunicación que hay entre los órganos del Estado, lo que genera impedimentos para realizar proyectos, como el propuesto en este trabajo de investigación, demostrando así una vez más que la "insularidad" de los agentes públicos generan problemas y los ciudadanos sufren las consecuencias.

Sin perder de vista que una ciudad inteligente va más allá de acumular proyectos académicos o mercadeo empresarial. Una smart city implementa acciones que mejoran la calidad de vida de sus habitantes por medios de las TIC's y una gestión que tiene por objetivo identificar aquellas problemáticas que entorpecen la vida de las personas (Cooper Hernández \& Vial Cossani, 2017) con el fin de darle una solución smart.

\section{Bibliografía}

Allan, J. R. (2001). Public-Private Partnership: A Review Of Literature and Practice.

Alvarado López, R. A. (2018). Ciudad inteligente y sostenible: Hacia un modelo de innovación inclusiva. PAAKAT: revista de tecnología y sociedad, 7(13). https://doi.org/10.18381/pk.a7n13.299

Azkuna, I. (2012). Smart Cities: Estudio internacional sobre la situacion de las Tic, la innovación y el conocimiento en las ciudades. Comisión de Ciudades Digitales y del Conocimiento, Bilbao, España. https://www.socinfo.es/contenido/seminarios/1404smartcities6/04BilbaoSmartcitiesstudy_es2012.pdf

Bardin, I. (1986). El análisis de contenido. Akal.

Berrone, P., \& Enric, J. (2019). Índice IESE Cities in Motion (Sexta). IESE Business School. https://media.iese.edu/research/pdfs/ST0509.pdf?_ga=2.69038738.2052512979.1566251906-1143235345.1566251906

BID. (2016). La ruta hacia las smart cities: Migrando de una gestión tradicional a la ciudad inteligente.

Bourdieu, P. (1980). Le capital Social. Actes de la Reacherche en Sciences Sociales.

Caragliu, A., Del Bo, C., \& Nijkamp, P. (2011). Smart Cities in Europe. Journal of Urban Technology, 18(2), 65-82. https://doi.org/10.1080/10630732.2011.601117

Casado, F. (2007). Alianzas público-privadas para el desarrollo. Fundacion Carolina.

CENSO. (2018). Síntesis De Resultado CENSO 2017. Insittuto Nacional de Estadisticas. Chile, 27.

CEPAL. (2019). Aunuario Estadístico de América Latina y el Caribe. https://repositorio.cepal.org/bitstream/handle/11362/44445/4/S1800772_mu.pdf

Chaparro, F. (2001). Conocimiento, aprendizaje y capital social como motor de desarrollo. Ciência da Informação, 30(1). http://revista.ibict.br/ciinf/article/view/935

Ching, T. Y. (2013). Smart cities: Concepts, perceptions and lessons for planners [Thesis, Massachusetts Institute of Technology]. https://dspace.mit.edu/handle/1721.1/81146 
Coe, A., Paquet, G., \& Roy, J. (2001). E-Governance and Smart Communities: A Social Learning Challenge. Social Science Computer Review, 19(1), 80-93.

https:// doi.org/10.1177/089443930101900107

Cohen, B. (2015, agosto 10). The 3 Generations Of Smart Cities. Fast Company. https://www.fastcompany.com/3047795/the-3-generations-of-smart-cities

Cohen, B., \& Obediente, E. (2014). ESTUDIO "RANKING DE CIUDADES INTELIGENTES EN CHILE". País digital, 77.

Cooper Hernández, D. C., \& Vial Cossani, C. (2017). Smart Cities en Chile: Entre la factibilidad tecnológica y las barreras políticas. Debates Latinoamericanos, 31, 10 a 19-10 19.

CORFO. (2018). Programa Estratégico Regional: Satiago Ciudad Inteligente. SeSantiago. https://www.dropbox.com/s/z01v0y8as7x53q1/RELATO\%20PROGRAMA\%20ESTRAT\% C3\%89GICO.pdf?dl=0

CORFO. (2019). Plan "Chile: Territorio Inteligente" de CORFO es reconocido en evento smart city de México - Sé Santiago. http://www.sesantiago.cl/plan-territorio-inteligente-de-corfo-es-reconocido-en-evento-smartcity-de-mexico/

Degiorgis, P. (2017). Smart Cities o Ciudades Inteligentes. Debates Latinoamericanos, 31, 1 a 2-1 2.

Devlin, R., \& Moguillansky, G. (2009). Alianzas público-privadas como estrategias nacionales de desarrollo a largo plazo. Revista de la CEPAL.

Díaz Herrera, C. (2018). Investigación cualitativa y análisis de contenido temátoco. Orientación intelectual de revista Uniersum. Ediciones Complutense.

Fernández González, M. (2016). La Smart City como imaginario socio-tecnológico. Cuadernos de Investigación Urbanistica, 109. https://doi.org/10.20868/ciur.2016.109.3498

Fundación Naciones unidas. (2003). Public-Private Partnerships: Meeting in the Middle, Washigton y Ginebra.

Giffinger, R., Fertner, C., Kramar, H., Kalasek, R., Milanović, N., \& Meijers, E. (2007).

Smart cities_-Ranking of European medium-sized cities. http://www.smart-cities.eu/download/smart_cities_final_report.pdf

Kliksberg, B. (2006). CAPITAL SOCIAL Y CULTURA, CLAVES DEL DESARROLLO. Cuadernos Latinoamericanos de Administración, II(2), 5-31.

López Noguero, F. (2002). El análisis de contenido como método de investigación. Revista de Educación, 4, 167-179.

Martínez, G. M. (2017). La agenda de las ciudades latinoamericanas en su camino hacia los ecosistemas de innovación. Debates Latinoamericanos, 31, 41 a 47-41 47.

Medina González, V., León Santos, M., \& Torres Ponjuán, D. (2015). La identificación del capital social en organizaciones de información y su relación con la gestión del conocimiento. 26(4), 424-436.

Montero, V., Muñoz, W., \& W. Simon, J. (Eds.). (2018). Transformaciones en la política y desafíos para gobernar en el siglo XXI. Universidad de Concepción.

Naciones Unidas. (2016). La Agenda 2030 y los Objetivos de Desarrollo Sostenible: Una oportunidad para América Latina y el Caribe. 93.

Odendaal, N. (2003). Information and communication technology and local governance: Understanding the difference between cities in developed and emerging economies. Computers, Environment and Urban Systems, 27, 585-607. https:// doi.org/10.1016/S0198-9715(03)00016-4

ONU. (2018). Las ciudades seguirán creciendo, sobre todo en los países en desarrollo. ONU DAES | Naciones Unidas Departamento de Asuntos Económicos y Sociales.

https://www.un.org/development/desa/es/news/population/2018-world-urbanizationprospects.html

Orellana, A. (2013). Gobiernos metropolitanos para Chile: La necesidad versus la factibilidad. Centro de Políticas Públicas UC, Temas de la Agenda Pública, 8(63). 
Pérez Jesús, M., \& Loro, S. (2013). Alianzas Público-Privadas para el Desarrollo Modelos para llevar a la práctica la Innovación Social Empresarial. CODESPA. http://www.mas-business.com/docs/alianzaspublico-privadas.pdf

Ramirez-Alujas, A. (2016). Carta Iberoamericana de Gobierno Abierto. CLAD: Centro Latinoamericano de Administración para el Desarrollo, 21.

Sábato, J., \& Botana, N. (1993). LA CIENCLA Y LA TECNOLOGÍA EN EL DESARROLLO FUTURO DE AMÉRICA LATINA. 11.

Salgado, A. (2007). Investigación cualitativa: Dieños, evaluación del rigor metodológico y retos.

Schettini, P., \& Cortazzo, I. (2015). ANÁLISIS DE DATOS CUALITATIVOS EN LA INVESTIGACIÓN SOCIAL. Universidad Nacional de la Plata, 120.

Relativo al fortalecimiento de la regionalización del país, no Boletin 7963-06 (2011). https://www.camara.cl/pley/pley_detalle.aspx?prmID=8381

Sikora - Fernández, D. (2017). Factores de desarrollo de las ciudades inteligentes. Revista Universitaria de Geografia, 26(1), 135-152.

SUBTEL. (2016). Séptima Encuesta de Acceso, Usos y Usuarios de Internet. https://www.subtel.gob.cl/wpcontent/uploads/2015/04/Informe-VII-Encuesta-de-Acceso-Usos-y-Usuarios-deInternet_VF.pdf

Teixeira Vitienes, J. A. (2017). Inteligencia urbana aplicada: Un legado necesario para la ciudad americana. Debates Latinoamericanos, 31, 111 a 113-111 113.

Villarejo-Galende, H. (2015). SMART CITIES: UNA APUESTA DE LA UNIÓN EUROPEA PARA MEJORAR LOS SERVICIOS PÚBLICOS URBANOS. Revista de Estudios Europeos, 66, 25-51.

Waissbluth, M. (2003). La Insularidad en la Gestión Pública Latinoamericana. CLAD: Centro Latinoamericano de Administración para el Desarrollo, 27, 7. 